\title{
Visualization-based Decision Tool for Urban Meteorological Modeling
}

\author{
Daniel G. Aliaga ${ }^{1} \quad$ Carlos Vanegas $^{1} \quad$ Ming Lei $^{2} \quad$ Dev Niyogi $^{2}$ \\ ${ }^{1}$ Department of Computer Science \\ ${ }^{2}$ Department of Earth and Atmospheric Sciences \\ Purdue University
}

\begin{abstract}
We present a visualization-based decision tool that enables exploring the link between urban land use and urban weather, in particular predicting and visualizing changes in urban temperature, precipitation, and humidity. Our work combines recent work from urban planning, weather and climate studies, and visualization and computer graphics. Our approach uses an interactive tool to quickly and automatically produce plausible detailed 3D city models by means of a hybrid computational simulation of urban behavior and procedural urban geometry. From the city model, urban morphology parameters are efficiently computed and used by our custom meteorological simulator which considers the influence of the urban landscape. The result is a compelling visualization ability for understanding the complex feedback between urban land use and the regional meteorology of current cities and of potential future cities with desired greening patterns. Our work includes a case study example spanning a $1600 \mathrm{~km}^{2}$ area.
\end{abstract}

\section{Introduction}

In this paper, we provide a visualization-driven decision tool composed of automatic 3D city model generation, urban morphology calculation, and an integrated meteorological simulation. Our work includes a case study example for the city of Indianapolis with prescribed land use change scenarios generated by our tool and the assessment of resulting impact on regional meteorology using a coupled numerical weather prediction model.

\subsection{Motivation}

Visualizing and assessing the interdependency of dense urban development and local weather is critical to a variety of stakeholders. Urban areas have a high population density and are important seats of socioeconomic activities. Further, as a result of buildings materials and human activities, urban areas are also typically warmer than the surrounding areas. This Urban Heat Island (UHI) phenomenon is well documented (Oke 1988) and has a variety of biophysical, ecological, energy, health, and behavioral impacts (Brunsdon et al. 2009, Eliasson et al. 2007). Urban areas can contribute to the warming of the regional climate (Fall et al. 2009), and thus developing "greener" buildings is being considered as one of the climate change mitigation strategies (Akbari et al. 2009).

Several planning and assessment operations consider urban structures and regional effects but are often performed manually and are time consuming. For example, several works have proposed case-specific landscape and urban planning strategies (Shashua-Bar et al. 2009), ecological concepts (Stremke and Koh 2010), and urban greening methodologies (Bowler et al. 2010, Conway 2009) that improve urban climate and urban life quality.

\subsection{Challenges}

Our work seeks to enable intuitively exploring "what if" scenarios of the complex feedback occurring between the urban space and the regional weather/ climate. Specif- ically, we address three challenges which have not been addressed by previous works in a cohesive and coherent framew ork and consequently have limited the integration of meteorological modeling with urban planning (Eliasson 2000). First, our work builds upon and extends urban modeling and simulation efforts. These systems are typically concerned either with explicit 3D reconstruction of an existing city from photographs or from LIDAR/ laser data, or with simulating the spatial distribution of streets, population, jobs, and other demographic data. The systems are not concerned with efficiently creating and changing 3D city models or with modeling the effects on the regional meteorology.

Second, we improve meteorological modeling systems by including urban land surface interactions in the simulation and by automatically computing the necessary urban morphology parameters. While a few meteorological systems support land surface models at high spatial resolution (e.g., 1 to 10 kilometers) and explicitly represent the urban land surface and the associated energy balance (e.g., Masson 2000), in all cases the computation of the urban morphology requires an a priori detailed 3D knowledge of the desired (future) urban space and often manually computes urban morphology parameters. In contrast, we combine our 3D city model exploration tool with automatic calculation of urban morphology values in order to easily calculate meteorological predictions.

Third, while the impact of the urban landscape on regional weather is well documented (Cotton et al. 2003, Niyogi et al. 2006 and 2010, Oke 1988, Shepherd et al. 2002), visualizations have usually been limited to strictly viewing, a posteriori, simulation data using map-based representations and choroplethic renderings of spatiallyvarying data. They do not exploit the visualization notion that users are comfortable with viewing and understanding 3D models of urban landscape and visualizations of meteorological effects. Even without inspecting up close 

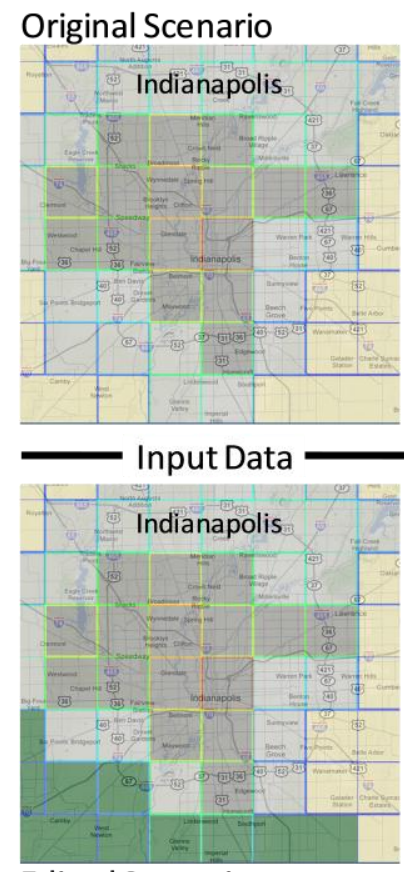

Edited Scenario

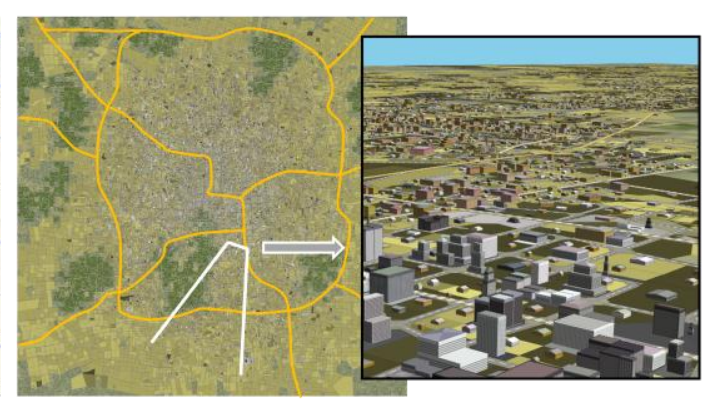

3D view

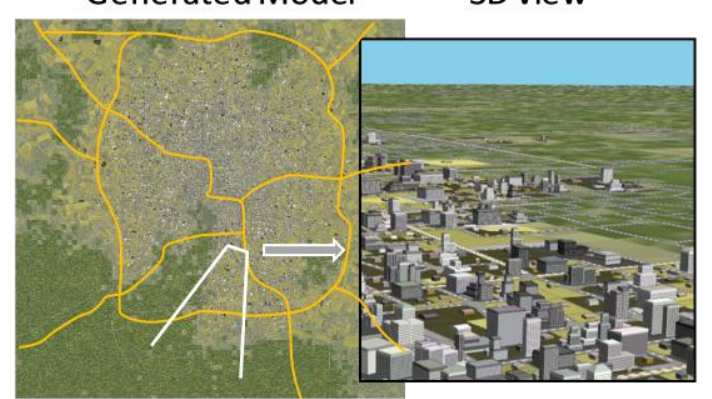

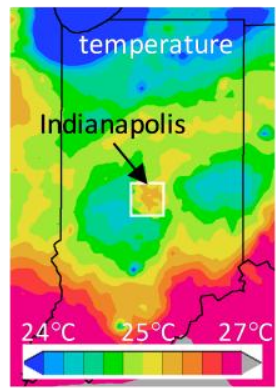

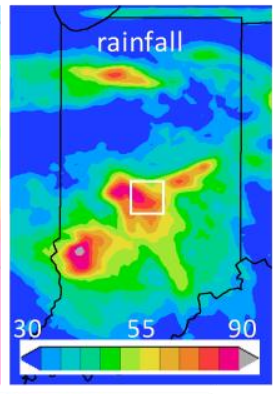

Simulated Weather Patterns $\rightarrow$
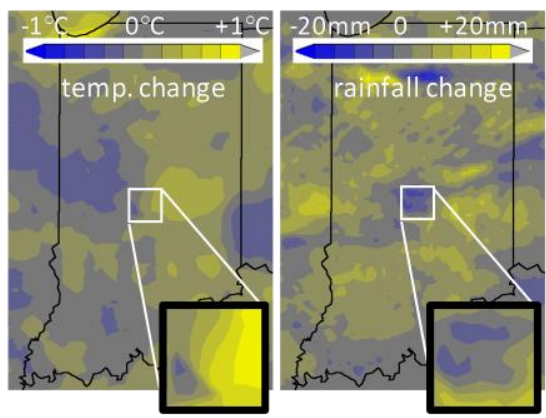

Figure 1. Visualization-based Decision Tool. Top: original urban scenario for Indianapolis, IN. Bottom: hypothetical (edited) urban scenario where the southwest corner became parks. Using LULC data (left column), complemented by population and terrain data, our tool automatically produces a plausible 3D city model (second and third columns) from which urban morphology parameters are extracted for a regional weather simulation over Indiana (fourth and rightmost columns). The ability to quickly edit the city model and automatically produce a plausible city and urban morphology parameters offers a substantial step forward in building integrated visualization and simulation systems for use in exploring policies for urban development and urban weather mitigation. In this example, the proposed change increases city temperature and decreases city rainfall.

and in detail, the overall pattern of streets, size of yards, mixture of buildings and green spaces, and amount of open space all contribute to providing an intuitive view of a place suitable for viewers of a wide range of expertise. By coupling automatic generation of 3D city models with an urban meteorological model, our tool provides a substantial step forward in building integrated and compelling visualization (and simulation) systems for use in dynamic urban land use / land cover (LULC) planning, urban weather patterns, and climate change mitigation.

\subsection{Overview}

Our visualization-based system enables assessing a novel urban scenario by automatically creating a 3D city model from LULC, population, and terrain elevation data, computing urban morphology values, and simulating the regional meteorological behavior. The typical usage cycle begins with an initial city model that can resemble an existing location or a desired future city. After viewing a city model and its resulting weather patterns, the user (or decision maker) can interactively alter the city model and repeat the cycle (see Figure 1; this figure will be further explained in the results section). Hence, urban planning policies and weather mitigation polices can be explored.

Our urban modeling methodology for creating a 3D city model and for computing the urban morphology parameters is to use an urban behavioral simulation process integrated with an urban geometry procedural generation process. This hybrid city-modeling process uses only a coarse specification of social and economic parameters of a city yet results in the automatic creation of a plausible 3D instantiation of the city. While the produced city model does not precisely recreate an existing urban area, it does produce a model of sufficient qualitative similarity to visualize the urban environment and to compute urban morphology parameters.

Our meteorological modeling system is based on the Regional Atmosphere Modeling System (RAMS version 4.3) (Cotton et al. 2003) coupled with an urban energy balance model-town energy budget (TEB) (Masson 2000). The system, together with the automatically computed urban morphology parameters considers the role of the urban land scape in predicted meteorological phenomena (e.g., storms and precipitation).

Altogether, we demonstrate our system in a case study example of over a $1600 \mathrm{~km}^{2}$ area centered on Indianapolis, IN (USA). We compare the results of a control scenario and various "what-if" greening scenarios with the objective of visualizing and understanding how to mitigate the local climate by different configurations.

\subsection{Impact and Contributions}

The impact and contributions of our work includes:

- a first system to dynamically integrate urban LULC planning and high impact/ extreme weather mitigation into a unified framework that enables stakeholders to explore the effects of adopting different urban land configurations, 
- a method to generate 3D city models from landuse, population, and terrain elevation data, and to interactively edit the city model assisted by an integrated urban simulation system to keep the model within a plausible configuration, and

- a set of algorithms to compute the urban morphology parameters necessary for regional weather simulation and visualization.

\section{Related Work}

Our research builds upon urban planning, simulation and visualization, meteorological modeling, and 3D urban modeling. Urban planning is of central importance in today's rapidly growing urban areas. Early works have considered the effect of urban planning on local climate. For example, Randall et al. (2003) proposed an extension to ArcGIS that enables the designer to "green" different parts of a city using one or more naturalization strategies. Adolphe et al. (2001) suggest simplified ways to compute indicators of urban morphology and to directly use those to plan for future urban climates. Recently, Shepherd et al. (2010) studied the effect of future urban growth on local weather for Houston, TX. However, the aforementioned systems provide a limited, if any, closed-loop system between urban modeling and urban weather simulation, and do not include automatic 3D urban modeling.

Urban simulation and visualization is traditionally used to help regional planning agencies evaluate alternative transportation investments, land use regulations, emergency response plans, and environmental protection policies (e.g., Terzi and Kaya 2011, Torrens and O'Sullivan 2001, Westervelt et al. 2011). A variety of entertainment applications also seek to simulate and grow plausible cities for use in games and in movies. Urban simulation models can be loosely divided into three dominant paradigms: cellular automata methods (Clark and Gaydos 1998, Al-kheder et al. 2008), agent-based methods (Portugali 2000), and urban-economic discrete choice models (De Palma et al. 2007, Vanegas et al. 2009a). Urban visualization often makes use of techniques including choroplethic maps generated by exporting simulation results, summarized by a zonal geography, to a GIS for rendering (e.g., Schwartzman and Borning 2007). Urban simulation and visualization does not typically address creating a 3D city nor does it focus on generating parameters for a meteorological simulation. Nevertheless, studies have shown that visualization helps in disseminating and communicating the results of a planning or simulation scenario (Laing et al. 2009, Pettit et al. 2011). Moreover, the improved communication can foment the adoption of environmentally-friendly planning (Brody et al. 2008).

Meteorological simulations that explicitly consider the effects of urban landscape are significantly different from simulations over other natural LULC. This is primarily due to the unique physical property of artificial materials and has drawn increased research interest in recent years (Akbari et al. 2009, McCarthy et al. 2010, Masson 2000, Oleson et al. 2010). The Urban Heat Island is caused by the high heat capacity and low albedo of concrete build-
Provide LULC, Population, Terrain Data

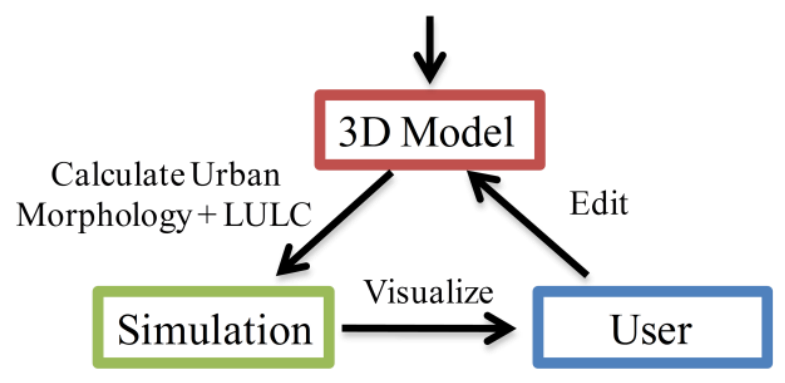

Figure 2. System Pipeline. A summary of our system pipeline. Given LULC, population, and terrain elevation data, we create an initial city model. Using the city model, we compute urban morphology parameters and new LULC/population data (if applicable). The results are fed to our urban weather simulation and then visualized by the user. The user can then perform highlevel editing operations on the model and repeat the cycle.

ings. Tall buildings also lead to the higher roughness and displacement length that alters regional surface winds and the atmospheric boundary layer convergence/ divergence patterns. Moreover, urban landscape generally has lower evaporation/transpiration or latent heat flux as compared to the surrounding rural region. This creates spatial gradients in surface heat fluxes, which combined with changes in boundary layer convergence can create zones of preferential convection, and mesoscale weather patterns and climatic regimes (Niyogi et al. 2010). Most urban weather simulation methods assume either a very simple urban geometrical model or offload all modeling to time consuming manual efforts (Niyogi et al. 2006).

$3 D$ urban modeling methods have been proposed to create detailed geometric models of cities and of buildings. Parish and Mueller's (2001) pioneering method created city models using a grammar-based approach. Such an approach has been extended to the generation of buildings and road networks (e.g., Aliaga et al. 2008, Chen et al. 2008, Lipp et al. 2008). However, none of these efforts have investigated effects on regional meteorology.

Recently, several research works have proposed an interdisciplinary collaboration between visualization and urban-related simulations to produce new modeling techniques. These techniques facilitate an intuitive presentation and increase the impact of urban simulation to many stakeholders. For example, Weber et al. (2009) describe a geometrical simulation system that models the plausible growth of a 3D urban model over time, based on an exogenous population model. Vanegas et al. (2009a) provide a visualization system that uses the output of a temporal agent-based urban simulation to make plausible images of future road and parcel networks. Vanegas et al. 2009b merge behavioral and geometrical modeling of urban areas in order to reduce the design time of 3D city models. However, in these works the focus is on designing and editing a new urban model, and not on modeling or assessing the meteorological impact of a current or altered city. Therefore, these systems do not produce any indicators for climatic patterns, do not support creating 

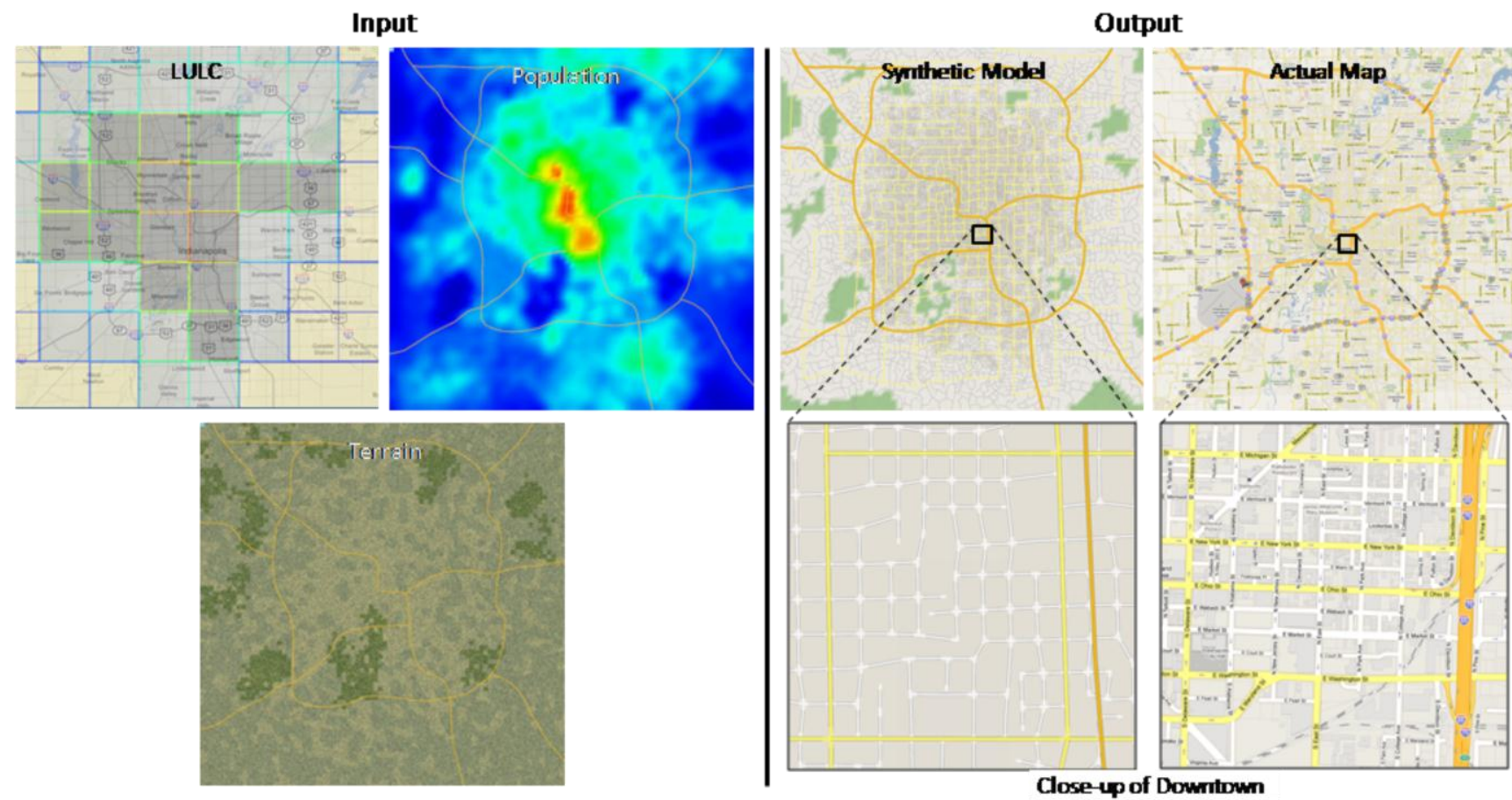

Figure 3. Urban Geometry Generation. On the left, we show LULC, population, and terrain specification (e.g., elevation, parks, water bodies) used to generate a plausible 3D city model. On the right, we compare the generated synthetic model to an actual map of Indianapolis (from Google Maps). Our system generates geometry that although not identical to the actual city is qualitatively similar. Note: since highways are not generated by a very predictable and/or understood process, we specify highways by hand.

3D models from LULC database, and do not provide customized tools for interactive editing of behavioral and geometric variables that affect meteorological processes, as is undertaken in the present study.

\section{Urban Weather Decision Tool}

Our system enables cycling through the process of creating/ editing a 3D city model, automatically generating parameter values for a meteorological simulation and then simulating urban weather over a short time horizon (e.g., a few days). Figure 2 provides an outline of our computational pipeline. The initial city model is created using LULC data, population/ jobs data (e.g., census information), and terrain elevation data. Our system then computes urban morphology parameter values suitable for our urban weather simulation. The resulting simulation values and city model are visualized by the user (e.g., $3 \mathrm{D}$ renderings, choroplethic maps, etc.). The user can then interactively change the city model using high-level editing tools. New urban morphology parameter values are computed and the cycle repeats enabling the exploration and visualization of new city designs and the resulting urban weather.

To support the various high-level city editing operations, we enable easy alteration of the LULC, population/ jobs, terrain, and other input values. When exploring alternative city designs, we are interested in large scale policy-relevant changes such as "greening the city", "placing parks throughout the city", "enforcing population/ housing growth into certain areas", and "altering the shape of the urban contour with the surrounding nonurban land". Meanwhile, our automatic city model generation ensures a sufficiently accurate and plausible 3D model is created for use in the weather simulation.

Our editing interface uses a paint-brush style tool to alter the aforementioned input values. For example, to green a zone, the user selects the "greening" brush and clicks on the area where greening should occur. The radius and intensity of the brush can be controlled to determine, in this particular case, the area of the greening zone and the density of the greening. This operation corresponds to setting the grid cells inside the brush to vegetation-covered locations. Similarly, terrain editing implies varying the elevation of the terrain and, in some cases, indicating the affected grid cells are covered by water bodies. Population relocation corresponds to moving developed zones covered with urban infrastructure.

\section{Urban Geometry and Morphology}

In the following, we describe how a geometrical model of a target city is efficiently generated and how urban morphology parameter values are estimated from the automatically produced city model.

\subsection{Geometry Generation}

Our generation process creates a $3 \mathrm{D}$ city model that resembles a chosen city and is suitable for urban morphology estimation (Figure 3). While airborne and terrestrial scanned cities are available, obtaining such scans is a very large effort. Moreover, the resulting models usually consist of a large collection of unstructured polygons. Hence, although the models contain more geometric details than needed for an urban weather simulation (i.e., the canyon model), there is no relation to the underlying urban attribute layers. This hinders their use in urban weather simulations and in high-level model editing. In- 
stead, we seek to require only a relatively sparse amount of input and to support using our system in a large number of cities, even if a 3D scan is not available.

Our approach is to integrate the spatially-varying data layers of LULC, population, and terrain elevation which are typically available in extensive GIS and GISlike data sources though not necessarily at the same resolution. The widespread availability implies our system can be used for many cities worldwide. The generation process consists of two main components.

- First, the spatially-varying input data layers are translated into spatial distributions of urban land surface variables. Population and terrain elevation layers are directly read into their respective simulation layers, while the LULC layer is analyzed by cases and used to modify several simulation variables.

- Second, a hybrid of urban simulation and procedural modeling is used to interactively compute plausible $3 \mathrm{D}$ city geometry. Our system includes an extension of previous procedural modeling software (Vanegas et al. 2009b), which includes an interactive version of the well-known UrbanSim softw are (Waddell 2002).

\section{Input Layers}

The spatially-varying input data layers (i.e., LULC, population, and terrain elevation) are used to define values for the spatially-varying variables of an urban simulation system, such as population, jobs, accessibility, vegetation, and water.

- LULC layer: This layer describes the cover types of the terrain (e.g., urban, forested, shrub land, agriculture). It is represented as a grid data structure. Each grid cell contains a vector of 31 values, each in the range $[0,1]$. The $i$-th component of grid cell's vector is the portion of the grid cell that is covered by the $i$-th cover type. A subset of these 31 cover types is implemented in our current system. In particular, the cover types for dirt and short/ tall grass are interpreted as terrain with no vegetation. Broadleaf cover types are interpreted as terrain with vegetation. Water cover type, when combined with appropriate elevation data, is used to specify a water body. Urban cover type is interpreted as there being a non-zero amount of population.

- Population layer: This layer contains the spatial distribution of population. It is used to compute the population and jobs layers of the urban simulation. The number of jobs is estimated based on the grid cell population and on the distance to the city center.

- Terrain elevation layer: Digital elevation models of the studied area are used to create the terrain and to outline water bodies.

The resolution of the aforementioned layers is in general different due to the nature of the coupled land - atmosphere modeling systems that seek to capture atmospheric motions from continental $(\sim 1000 \mathrm{~km})$ to urban scale $(\sim 0.1 \mathrm{~km}$ or finer). In our case, LULC and population grid cells are typically available at coarse (e.g., $5 \mathrm{~km})$ resolution. In contrast, elevation data is denser $(\sim 0.1 \mathrm{~km}$ or finer). Further, the urban simulation system supports a dense grid (e.g., 100x100m). Hence, interpolation is used to combine the layers stored at different resolutions.

\section{D City Model}

Our city modeling component extends that of Vanegas et al. 2009b to the specific requirements of meteorological simulation. While Vanegas et al. 2009b use the generated output to increase the detail/ precision of predictive urban simulation, we use it for meteorological simulation. We focus on i) scenario editing (as described in Section 3) and on

- modeling from GIS data input - we customized the system so that an initial 3D model can be generated based only on the data sets that are normally available for weather/ climate simulation (i.e., LULC, population); nevertheless, additional layers can be provided to the system to improve the resulting model, either from databases or through user editing; and

- data generation for meteorological simulation - we automatically compute indicator values, in standard formats, from the generated 3D geometry to be given to the weather simulation system (Sections 4.2 and 5); further, our system generates significantly fewer polygons than in Vanegas et al. 2009b and only to the extent relevant to meteorological simulation, in order to more efficiently support large real-w orld cities.

Our use of the 3D city modeling engine of Vanegas et al. $2009 \mathrm{~b}$ produces geometrical models of cities from the aforementioned spatially-varying input variables (e.g., LULC, population, and terrain elevation) and from other interactively specified variables such as road length per grid cell, average road tortuosity, and building volume. A plausible set of interdependencies between the variables is determined from urban simulation models and literature. These variables are then fed into an iterative dynamical system which attempts to find a set of values that define a state of equilibrium; i.e. where the demands of behavioral modeling match those of geometrical modeling and vice versa. For example, given a population and job count, the total building volume necessary to house the population and jobs is computed. Then, building footprints and building envelopes are computed to match the calculated target building volume.

While methodologies for solving iterative dynamical system are well-known, the challenge is in providing expressions to evaluate the variable values and their derivatives. The city modeling engine uses algorithms (or symbolic equations when possible) for efficiently computing each of the described variables and their derivatives. Behavioral variables are computed by means of an agentbased simulation (e.g., population, jobs) or spatiallydefined metrics (e.g., accessibility, land value). Geometric variables are computed by means of 3D procedural modeling (e.g., road length, tortuosity). Results in Vanegas et al. $2009 \mathrm{~b}$ show that the solution finding process appears to be stable. Also, constraining variables to be within reasonable values as well as small step sizes ensures a minimal amount of oscillations during solution finding.

The experiments shown in Figure 3 (right half) and the similar validation Figure 8 in Vanegas et al. [2009b] 


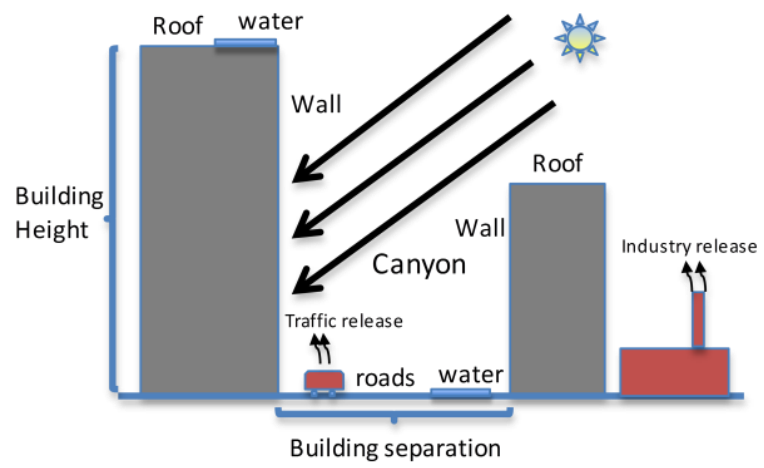

Figure 4. Canyon Model. The urban canyon model uses information such as building height, building separation, road width, road length, and other factors to estimate the interaction between urban structures and the atmospheric model.

demonstrate the ability to recreate plausible approximations of existing locations from a previously specified set of behavioral and geometrical variables. In both figures, terrain elevation, the centerline of major highways, parks, downtown location, and total population and jobs count are provided. As can be observed, the resulting synthetic 3D model is similar visually and in terms of substantive attributes, but clearly, the synthetic model will not match perfectly in all details. However, the similarity in structure and spatial distribution provides sufficient plausible 3D information for feeding into our later described meteorological simulation.

\subsection{Morphology Estimation}

The meteorological simulation takes as input a set of urban morphology values describing key geometric attributes of the city. Most previous urban weather simulations use a simple slab model whereby urban and nonurban areas are differentiated merely by a "type" field. Previous methods that do use some form of urban morphology often generate the values by hand or through a static database (e.g., Ching et al. 2009), with much effort, for a single city or fraction thereof. In our approach, the morphology data is directly computed from the previously described 3D model of the city. The advantages of this are an increased level of accuracy and automaticity.

Our main urban morphology parameters are:

- average building height: our system computes an area-weighted sum of heights of buildings in each grid cell, divided by the building count.

- urban fabric covered by buildings: our method sums up of the areas of the building footprints, $\mathrm{di}$ vided by the total area covered by structures (i.e., buildings and roads).

- canyon aspect ratio: this parameter is computed as the average ratio between building height and building to building-across-the-street distance, for all buildings in a grid cell.

- roughness and displacement length: this value estimates the surface roughness of an urban area (e.g., a measure of how much the actual roof/ road geometry varies from planarity as well as the roughness of the roof/ road material). This value could be computed as the standard deviation of the buildings height and road elevation in a grid cell plus another empirical surface roughness constant. In our examples, since terrain is often relatively flat within the city, the roughness is dominated by the variation in building heights within a grid cell. We typically use an empirically determined fraction of the building height (e.g., $1 / 10$ of the building height).

- albedo: this value primarily depends on the road/ roof material used; by default we use an empirical constant used to mimic stand ard roads and roofs. Nevertheless, an "albedo brush" is easily available to alter the value.

- internal building temperature: this value could be user-specified but in our example scenario we assume a constant temperature of $17^{\circ} \mathrm{C}$.

It is worth noting that the urban morphology parameters are re-estimated after any editing operation or change of values in the input layers. Similarly, after editing operations that modify the city's configuration (e.g., "greening", "placing parks", "growing housing"), LULC data is also updated. The computed values for urban morphology and LULC are saved in a temporary file that is read by the urban weather simulation component. The file size varies with the size and resolution of the grid, and with the number of LULC parameters that are considered, and $\mathrm{w}$ as typically under $1 \mathrm{MB}$ in our examples.

\section{Urban Meteorological Simulation}

Exploring, simulating, and visualizing urban weather patterns hinges on coupling the urban geometrical surface and the atmosphere and on modeling the resulting fluxes in meso-scale atmospheric models. This is a challenging problem due to the complexity and diversity of cities around the world. In general, a regional weather simulation consists of a large-scale atmospheric model integrated with a land-surface interaction model. When including urban areas, the interaction with the urban structures must also be considered. Two main approaches have been followed: i) building-resolving models (BRM) represent individual building shapes and allow for detailed examination of specific processes (e.g., radiative effects, wind channeling, etc.) (Teemusk and Mander 2010), but BRM is inherently computationally-intensive which prohibits extending the simulation to an entire city, and ii) a simpler approach which relies on a "canyon" model (Oke 1988) where roads and building walls collectively form a set of canyons. This latter approach is more adept at modeling cities with large horizontal sizes and is the methodology used in our work.

Based on the canyon model, we adapt and extend the Town Energy Budget (TEB) scheme of Masson (2000) to simulate the surface energy fluxes into the atmosphere at the surface of a mesoscale atmospheric model covered by buildings, roads, and other artificial material. The fluxes estimated from TEB are combined with those of other surrounding land types (e.g., vegetation, water bodies, etc.) using the LEAF2 land-surface model and then spatially averaged into a single atmospheric model grid in proportion to the space occupied by each land type. The 
overall atmospheric simulation system we use is the RAMS version 4.3 (Cotton et al. 2003). RAMS is a welltested multi-scale environmental modeling system, and has been applied to a wide range of problems (e.g. flow around buildings, thunderstorm dynamics, regional circulations, and continental scale climate change analysis).

Our implementation addresses the challenge of integration of fine scale data, needed to accurately represent the urban landscape morphology, and coarser scale computational and theoretical requirements of the formulations used within numerical weather prediction (NVP) models (i.e., RAMS). For instance, urban morphology expects $1 \mathrm{~m}$ resolution while the NWP model grid spacing is typically around $1 \mathrm{~km}$. To blend these heterogeneous scales, a possible approach -- and the one adopted in this study -- is to use a subgrid scale representation of the land scape 'tiles' within the larger grid. The patchwork of different land uses within a model grid are aggregated to form a single 'tile'. The four most prominent landscapes in a grid (i.e., from a choice of International GeosphereBiosphere Programme land use categories such as mixed forest, shrublands, mixed agriculture, deciduous forest, urban, etc.), and the urban region (if not already part of the top four land use fraction in that grid) are tiled together to form the grid-based landuse representation. The model equations for energy and hydrological balance are then solved for each tile and then area-weighed to develop the grid averaged value. Following Avissar and Pielke (1989), a subgrid scale flux correction is also made to account for the tiling heterogeneity.

Specific within the LEAF2 urban tile, the leaf area index and fractional vegetation coverage are decreased while the aerodynamic roughness length is increased to approximate hydrodynamic effects. In coupling with TEB, the generalized canyon model replaces the LEAF2 urban class parameterization to better represent the urban processes. The 3D urban surface and roughness sub-layer interactions required for solving the surface layer feedbacks in the mesoscale model and the input data for TEB is imported from the 3D visualization model. TEB provides the lower boundary conditions required for the atmospheric model to respond to and the simulated forecast for surface quantities including sensible heat flux, latent heat flux, momentum changes, albedo, and emissivity. RAMS imported TEB's fluxes for each grid cell at the first atmospheric level as an urban patch contribution, then average with LEAF2 nonurban patches are translated into soil moisture/ temperature changes, and air temperature, humidity and winds following surface layer similarity approaches (Arya 1988). The total (grid averaged) fluxes and scalar output for the grid cells with u rban land use consist of both TEB and LEAF2 values.

Figure 4 depicts the geometrical configuration used by the TEB. The main shape dimensions include values for road length, road width, building separation, and building height. The TEB uses and computes three surfaces temperatures: one for each of roofs, roads, and walls. For each surface type, one or more layers are present - this enables modeling the fluxes to/ from building interiors (e.g., walls and roofs) or to/ from the ground (e.g., roads).
Further, since surface temperatures are included in the model, it is possible to model the evaporation of water accumulated from precipitation. The TEB assumes surfaces to be impervious and lets water evaporate as long as the air humidity is unsaturated and there is still water on the surfaces. In addition, the TEB accounts for solar radiation trapped inside the urban structures by considering how much of the sky is visible from the walls/ streets (roofs always have full view of the sky). Finally, the TEB also considers anthropogenic fluxes (i.e., fluxes due to human activity) which primarily consist of domestic heating and combustion.

\section{Implementation Details}

Our geometry generation system, urban morphology estimation, and model editing tools run on a desktop PC with a $3.0 \mathrm{GHz}$ processor and $4 \mathrm{~GB}$ memory. Our urban modeling system is an extension of the infrastructure described in Vanegas et al. 2009b (available upon request from authors). All rendering is done on the PC using an NVidia Quadro FX 1700 graphics card. The compute time for city generation and for all visualizations ranges from seconds to minutes.

The regional weather simulation is based on the RAMS system [Cotton et al. 2003] (available at http:// bridge.atmet.org/ users/ software.php, version 4.3). The simulator runs on a cluster using up to 5120 cores (640 nodes of 32GB memory, each node with $82.5 \mathrm{GHz}$ cores, and interconnected by 10 gigabit Ethernet). The clusterand PC-based portions of our system communicate using temporary files. The offline simulation compute time depends on the total region size and the grid sizes of the simulated area; i.e., time-accuracy tradeoffs can be made. In our experiments, we need 6 to 24 hours for a simulated time of 3 days depending on the grid resolution, process calculations and representations, and system efficiency.

Our example case-study focuses on the Indianapolis, IN, USA metropolitan area. This area spans approximately 50 by 45 kilometers and has nearly two million people. To make the simulation practical on our cluster, our highest level-of-detail regional weather simulation was configured with three telescopic nested grids with 80,20 , and $5 \mathrm{~km}$ grid spacing with a common center point at $39.77 \mathrm{~N}$ and 86.16W (i.e., downtown Indianapolis). Grid 1 had 64 $\mathrm{x} 48$ horizontal grid points covering the entire continental United States (US) with a prognostic time step of 90 seconds. The second grid had $82 \times 74$ horizontal grid points covering most of the Eastern part of the US with a time step of 30 seconds. The third grid comprised of $94 \times 94$ horizontal grid points with a time step of 10 seconds, and covers all of Indiana. The model was configured based on success with previous cases documented in Lei et al. (2008) and Lei and Niyogi (2011) and with terrain following the pressure/ sigma-coordinate system with 36 vertical layers with a finer vertical spacing from $0.05 \mathrm{~km}$ to $1.27 \mathrm{~km}$ and a fixed spacing thereafter until $8.5 \mathrm{~km}$, and a total depth of the model atmosphere set to $21 \mathrm{~km}$. The TEB was coupled only over the inner most region of grid 3 which covers the Indianapolis urban area. Urban energy 


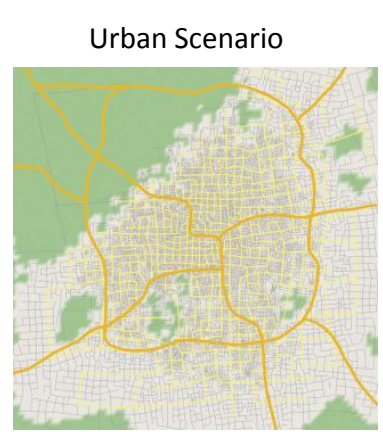

NW greening

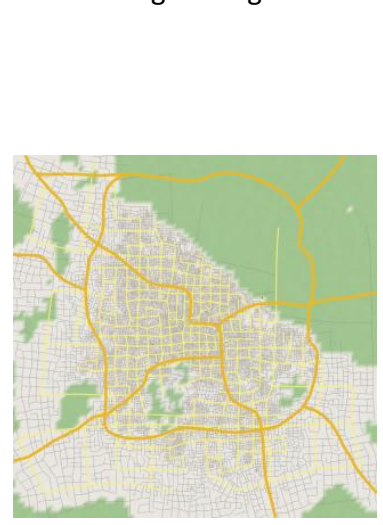

NE greening

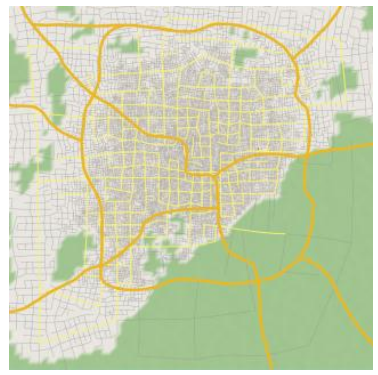

SE greening
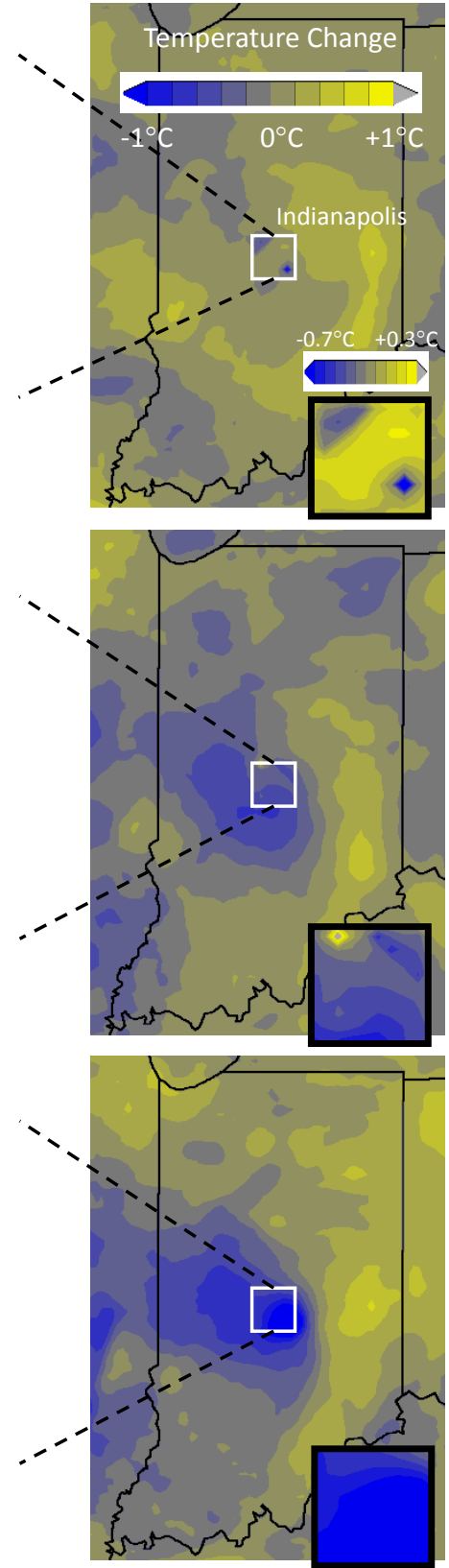
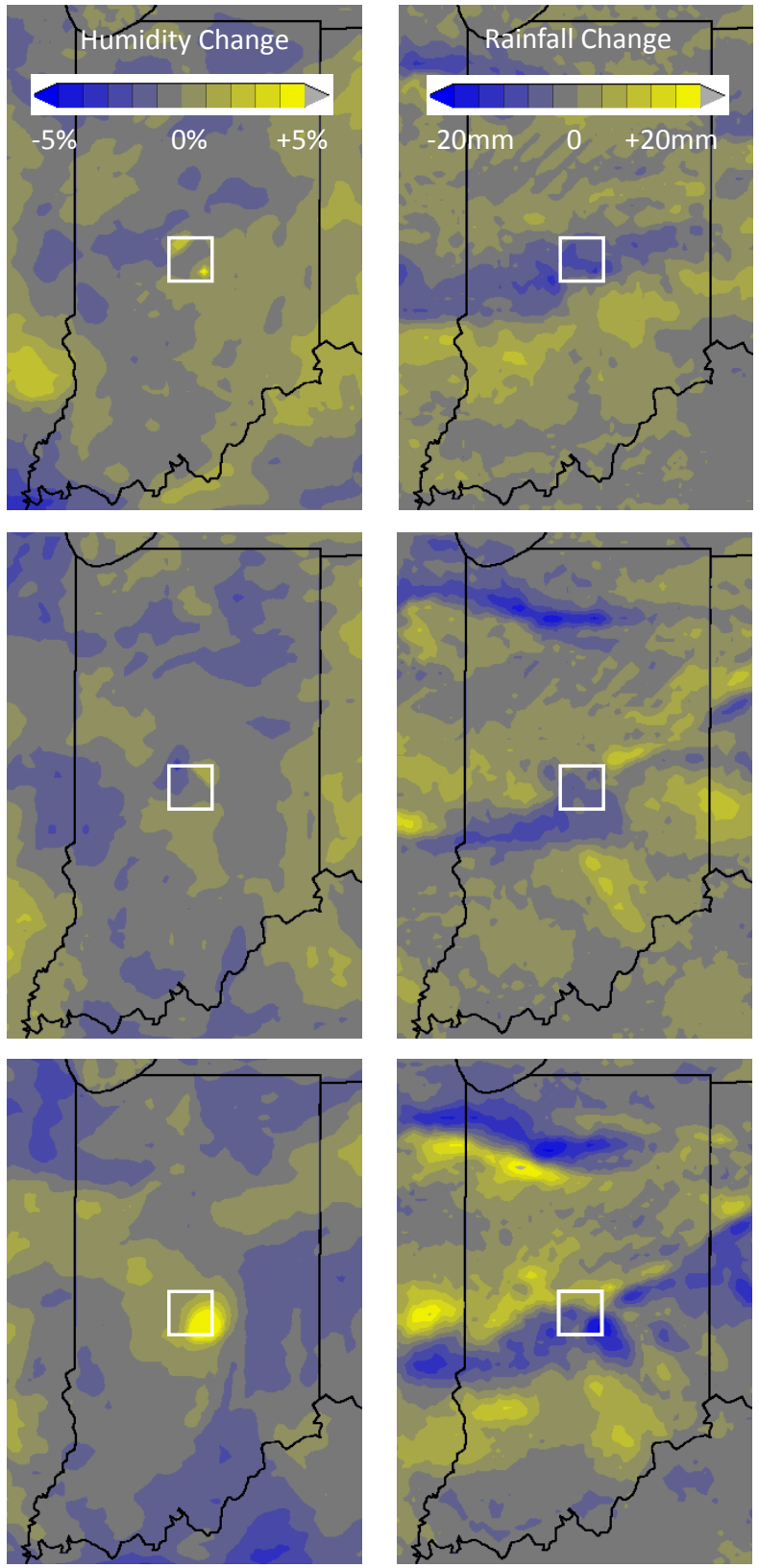

Figure 5. Visualizations of Urban Weather Mitigation. Visualization of greening the NW (top row), NE (middle row), or SE (bottom row) corner of Indianapolis. The first column shows a map-style view of the automatically generated 3D model produced by our system. The second, third, and fourth columns show the change in temperature, humidity, and rainfall as compared to the control scenario. Close-ups are provided of the temperature change over Indianapolis.

balance was invoked on the grid either with TEB (for grid 3) or a simpler slab model for the outer two domains.

The input layer data was obtained from county and GIS databases: LULC data came from U.S. Geological Survey and the population data is from year 2000 census. The simulation model was initialized using NOAA Final Analysis (FNL) data, which were also used for providing the lateral boundary conditions every 12 hours for the outermost domain.

For additional information about system details and/ or copy of the datasets, please contact the authors.

\section{Example Results}

In this section, we explore several altered urban scenarios of the region surrounding Indianapolis, IN with different greening configurations. Recent investigations have shown that varying the green areas (Clark et al. 2010, Conway 2009, DeNardo et al. 2005, Oberndorfer et al. 2007) and the geometry and material of urban area buildings (Teemusk and Mander 2010) can significantly alter temperature, humidity, and rainfall, for example. Using our system, decision makers can view and explore tentative city models as well as choroplethic maps of the weather. In our examples and during the simulation time 


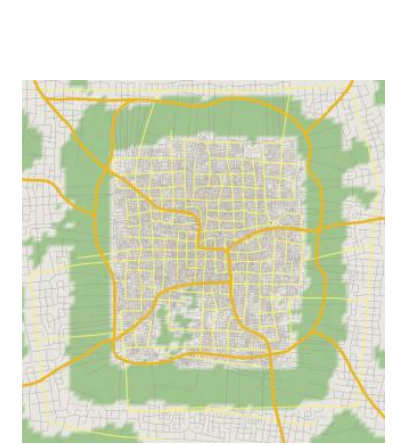

Circular Greening

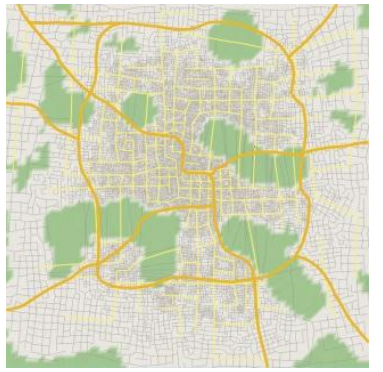

Distributed Greening
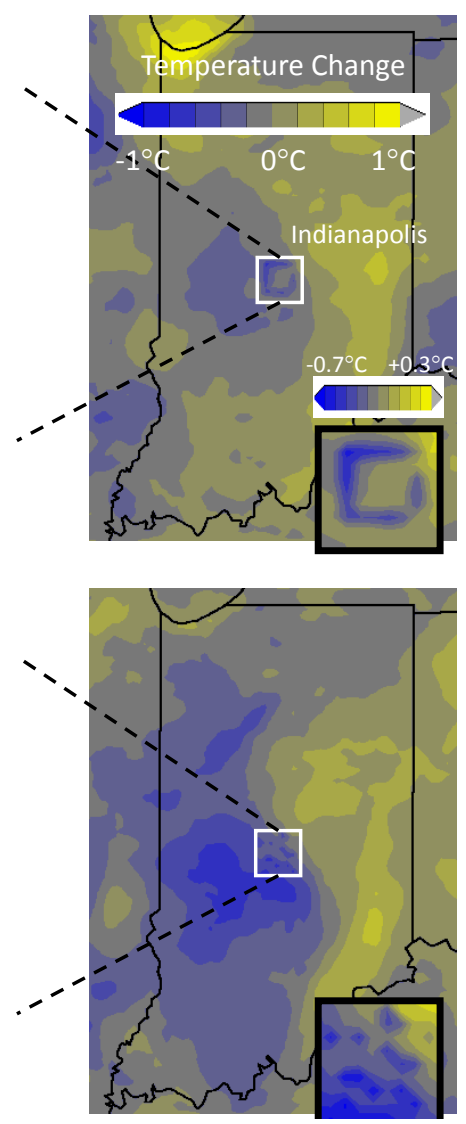
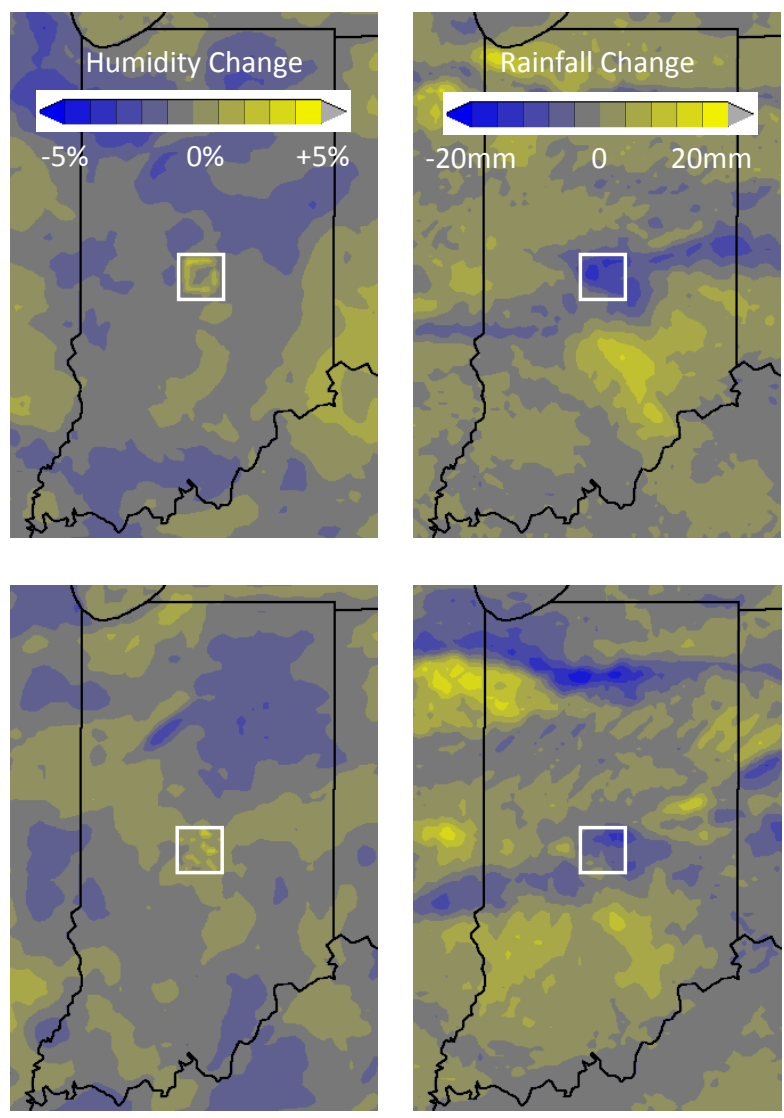

Figure 6. Visualization of Tentative Urban Policies. These example images extend the scenarios of the previous figure by the enforcing tentative urban policies: e.g., a ring of parks around the city (top-row) or parks within the city (bottom-row).

frame, the surrounding regional weather (e.g., from state and country level meteorological phenomena) is producing southwest winds over Indianapolis (e.g., winds from the southwest and to the northeast). A region that receives the winds first is said to be "upwind" and region that receives it second (or later) is called "downwind". As a control simulation, we perform a 3-day urban weather simulation over the current configuration of Indianapolis. The base-levels of temperature, rainfall, and humidity of this control scenario are shown in the two upperrightmost images of Figure 1 and in the left-most image in Figure 7.

A first explorative scenario is to add parks over the southwest (SW) corner of Indianapolis (Figure 1). The SW corner is upwind and hence is the first part of the metropolitan area hit by incoming regional weather activity (e.g., by a thunderstorm) from the larger surrounding area. To create this scenario (and all other scenarios), we use our interactive system to perform a high-level greening operation by changing the LULC for the selected region to parks using our GUI and then the system automatically redistributes the affected population to elsewhere in the city and generates a plausible new set of streets and building structures.

Figure 1 shows views of the generated 3D city and colored imagery of the simulation results. These results show the expected temperature decrease over the SW corner. Research studies of partial greening (e.g., near $50 \%$ greening of roof tops in New York City (Rosenzweig et al. 2006), similar greening in subsets of Toronto, Ontario (Banting et al. 2005), addition of parks as studied by Bowler et al. (2010)) has showed temperature reductions of $0.8^{\circ} \mathrm{C}$ to $2^{\circ} \mathrm{C}$, which are similar in range to our results. However, due to the feedback on other weather variables almost all other areas over downwind Indianapolis become warmer in this case. In addition, there is less rainfall over Indianapolis but noticeably more rainfall further downwind from Indianapolis (i.e., over the NE corner outside of Indianapolis). It has been observed that the cooling effects of green areas within a city can affect weather far beyond the boundary of the green area itself (Honjo and Takakura 1991). Thus, in general green areas have lower temperatures and higher humidity as compared to dense urban (cement) areas. But the weather resulting from large green areas can affect not only other parts in the city but also surrounding non-urban areas (Lei and Niyogi 2011). Hence, the temperature/ humidity/ rainfall changes that are observed are typical of regional meteorological simulations and are not model artifacts. Our system assists in predicting the general tendencies that are most likely under the provided initial conditions (Shepherd et al. 2010). In summary, our first scenario increases the average city temperature, decreases rainfall over Indianapolis, and increases rainfall over the NE corner outside of Ind ianapolis.

In this second experiment, we add some parks over the northwest (NW) corner of Indianapolis (Figure 5, toprow). Similar to the first experiment, the temperature de- 


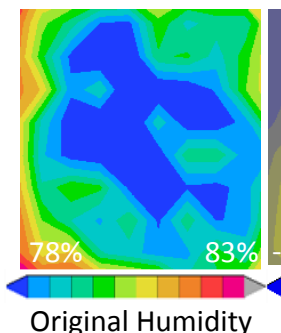

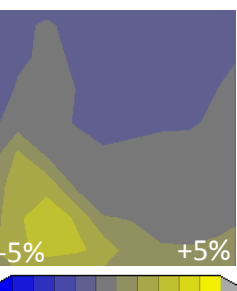

SW

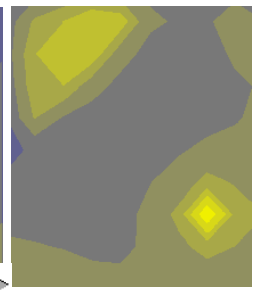

NW

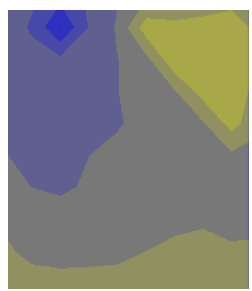

NE

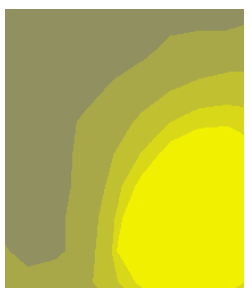

SE

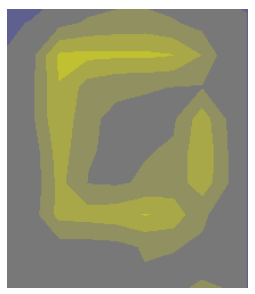

Ring

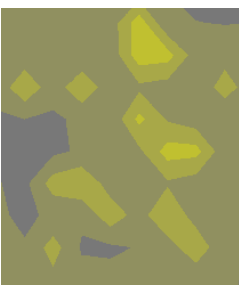

Distributed

Figure 7. Example Comparison. A visualization of the effect on humidity over Indianapolis for the different scenarios. (left) The original humidity level. Each picture to the right is the relative humidity level to the original scenario for the greening scenarios of $\{$ SW, NW, NE, SE, ring, distributed \} parks. SW-scenario produces the least overall humidity and SE-scenario the most overall humidity.

creases and humidity increases over the NW corner. But in this scenario, the average temperature over Indianap olis increases less than in the first scenario, the rainfall over Indianapolis is even less than in the first scenario, and almost no increase of rainfall occurs over the NE corner outside of Indianapolis. Given an objective of discovering a city configuration with no significant average temperature change and less rainfall, this scenario is a suitable one.

In a third and fourth experiment, we add some parks either over the northeast (NE) corner (Figure 5, middle row) or of the southeast (SE) corner (Figure 5, bottom row) of Indianapolis. In both of these scenarios, we observe both the temperature and the rainfall over Indianapolis generally decrease. Moreover, for the greening of the SE corner the temperature decreases the most while for the greening of the NE corner the least overall rainfall is produced, especially in the upper north half of the city. Thus, if the desire is to decrease temperature and rainfall over Indianapolis, these configurations provide options with different tradeoffs. The SE greening option provides the largest temperature decrease (of about $1^{\circ} \mathrm{C}$ ) because of a successful use of greening combined with the wind pattern. It is worth nothing that for a more drastic temperature reduction, it has been shown that more extensive greening of the urban canyon (Alexandri and Jones 2008) can have peak temperature reductions nearing $8.4^{\circ} \mathrm{C}$. Indeed for each of these options, longer term simulations (e.g., seasonal to multi-year) will need to be performed and the current examples are simply for illustrative purposes.

In Figure 6, we provide two additional urban scenarios. These are similar to those of the Figure 5 but could be viewed as the result of adopting an urban development policy. Figure 6-top ensures the city is surrounded by parks, while Figure 6-bottom enforces a policy of placing large parks distributed within the city. Both of these urban scenarios actually yield the most reduction in rainfall, as compared to the examples in Figure 5, as well as a mild temperature reduction on average. On the one hand, the introduction of parks throughout the city reduces the temperature the most. On the other hand, the ring of surrounding parks, decreases rainfall the most.

Finally, Figure 7 succinctly visualizes the change in humidity as a consequence of the different greening scenarios. The humidity increase over the parks is clear in most cases. The overall behavior of humidity can also be observed. This visualization could be complemented by views of the underlying 3D city model. Collectively, this yields an intuitive view of several "what-if scenarios" that would otherwise require significant manual (modeling and estimation) effort for our ensemble of experiments. This increase in humidity in response to greening is an important feature, which needs to be considered, for example, if the aim is to reduce thermal heat stress. A small reduction in temperature but large increase in humidity may actually significantly increase the heat/thermal stress, and cause more discomfort or bio-meteorological impacts (Kalkstein 2000).

\section{Discussion and Future Work}

We have introduced a first system that integrates automatic urban model generation and weather simulation into a single visualization system for exploring urban weather mitigation. Our work is important to visualization for it shows how tightly integrating intuitive visualizations and complex simulations into a closed loop can benefit the end-users. Our work is important to the application domain (e.g., urban planning, regional meteorology, and urban climate change mitigation) for it enables quickly exploring interaction setween cities and weather, potentially leading to improved schemes for urban weather modeling. Finally, our work is important to urban planning stakeholders, for it provides them with a compelling tool to explore the effect of different urban and regional LULC policies. While in our current system we focus on "greening effects", there is in principle no limitation on the type of changes to the urban environment. Our system is not intended to choose the best urban policies to accomplish a specified goal but is setup to en able their exploration.

With regards to future work, there are several items we wish to pursue. First, we would like to extend the number of urban morphology parameters and land use types that are explicitly supported. We will also test the approach over different urban geometries, and climate regimes (e.g. Atlanta, Houston, Mumbai/ India, etc.). Second, aside from a pure exploratory objective, we would also like to enable the system to automatically search for the least change to a current city that best accomplishes a desired goal; e.g., seek for an urban configuration producing lowest temperature and humidity, or producing most rainfall surround the city (where farmland is assumed to be). Third, we are investigating PC-based and GPU-based implementations of an approximate regional atmospheric 
modeling system to enable interactive simulation as well. Fourth, working with the State Climate Offices, we are actively seeking experimental deployments of our system.

\section{Acknowledgments}

We are grateful to our sponsors who enabled this work including NSF IIS 0964302, NSF OCI 0753116, and a Google Research gift.

\section{References}

[1] Adolphe L., 2001. A simplified model of urban morphology: application to an analysis of the environmental performance of cities. Environment and Planning B, 28, $183-200$.

[2] Akbari H., Menon S., Rosenfeld A., 2009. Global cooling: increasing world-wide urban albedos to offset CO2. Climatic Change, 94, 275-286, DOI: 10.1007/s10584-008-9515-9.

[3] Al-kheder S., Wang J., and Shan J., 2008. Fuzzy inference guided cellular automata urban-growth modeling using multitemporal satellite images. International Journal of Geographic Information Science, 22, 11-12, 1271-1293.

[4] Alexandri E. and Jones P., 2008. Temperature decreases in an urban canyon due to green $w$ alls and green roofs in diverse climates. Building and Environment, Part Special: Building Performance Simulation, 43(4), 480-493.

[5] Aliaga D. G., Vanegas C. A., and Benes B, 2008. Interactive example-based urban layout synthesis. ACM Transactions on Graphics 27, 5, 1-10.

[6] Arya, S.P., 1988. Introduction to micrometeorology, Academic Press, Inc. (International Geophysics Series, Volume 42), 324 p.

[7] Avissar, R. and Pielke, Sr., R. A., 1989. A parameterization of heterogeneous land surfaces for atmospheric numerical models and its impact on regional meteorology. Monthly Weather Review, 117, 2113-2136.

[8] Banting D., Doshi H., Li J., Missios P., Au A., Currie B.A. and Verrati M., 2005. Report on Environmental Benefits and Costs of Green Roof Technology for the City of Toronto. The City of Toronto and Ontario Centres of Excellence - Earth and Environmental Technologies (OCE-ETech).

[9] Bowler D., Buyung-Ali L., Knight T., Pullin A., 2010. Urban greening to cool towns and cities: A systematic review of the empirical evidence. Landscape and Urban Planning, 97(3), 147155.

[10] Brody S., Zahran S., Grover H., Vedlitz A., 2008. A spatial analysis of local climate change policy in the United States: Risk, stress, and opportunity. Landscape and Urban Planning, 87(1), 3341.

[11] Brunsdon C., Corcoran J., Higgs G., Ware A., 2009. The influence of weather on local geographical patterns of police calls for service. Environment and Planning B, 36, 906 - 926.

[12] Ching, J., M. Brown, S. Burian, F. Chen, R. Cionco, A. Hanna, T. Hultgren, T. McPherson, D. Sailor, H. Taha, and D. Williams, 2009. National Urban Database and Access Portal Tool. Bulletin of the A merican Meteorological Society, 90, 1157-1168.

[13] Chen G., Esch G., Wonka P., Mueller P., and Zhang E., 2008. Interactive procedural street modeling. ACM Transactions on Graphics 27, 3, 1-10.

[14] Clark C., Busiek B.E., and Adriaens P, 2010. Quantifying Thermal Impacts of Green Infrastructure: Review and Gaps. Water Environment Foundation (WEF) 2010 Cities of the Future/Urban River Restoration Conference, Cambridge, MA.

[15] Clarke K. C., and Gaydos L.J., 1998. Loose-coupling a cellular automaton model and GIS: long-term urban grow th prediction for San Francisco and Washington/Baltimore. International Journal of Geographical Information Science, 12(7), 699-714.
[16] Conway T., 2009. Local environmental impacts of alternative forms of residential development. Environment and Planning B, $36,927-943$

[17] Cotton, W. R., Pielke, Sr., R. A., Walko, R. L., Liston, G. E., Tremback, C. J., Jiang, H., McAnelly, R. L., Harrington, J. Y., Nicholls, M. E., Carrio, G. G., and McFadden, J. P., 2003. RAMS 2001: Current Status and Future Directions. Meteorology and Atmospherical Physics, 82, 5-29.

[18] De Palma A., Picard N., and Waddell P., 2007. Discrete choice models with capacity constraints: An empirical analysis of the housing market of the greater Paris region. Journal of Urban Economics, 62, 2, 204-230.

[19] DeNardo J.C., Jarrett A.R., Manbeck H.B., Beattie D.J., and Berghage R.D., 2005. Stormwater mitigation and surface temperature reduction by green roofs. Transactions of the ASABE, 48(4), 1491-1496.

[20] Eliasson I., 2000. The use of climate knowledge in urban planning. Landscape and Urban Planning, 48(1-2), 31-44.

[21] Eliasson I., Knez I., Westerberg U., Thorsson S., Lindberg F., 2007. Climate and behaviour in a Nordic city. Landscape and Urban Planning, 82(1-2), 72-84.

[22] Fall S., Niyogi D., Pielke Sr. R. A., Gluhovsky A., Kalnay E., and Rochon G., 2009. Impacts of land use land cover on temperature trends over the continental United States: assessment using the North American Regional Reanalysis. International Journal of Climatology, DOI: 10.1002/joc.1996.

[23] Honjo T. and Takakura T., 1990-91. Simulation of Thermal Effects of Urban Green Areas on their Surrounding Areas. Energy and Buildings, 15-16, 443.

[24] Kalkstein L., 2000. Saving lives during extreme weather in summer, British Medical Journal, $321: 650$.

[25] Laing R., Davies A.M., Miller D., Conniff A., Scott S., Morrice J., 2009. The application of visual environmental economics in the study of public preference and urban greenspace. Environment and Planning B, 36, $355-375$.

[26] Lei M., Niyogi D., Kishtawal C., Pielke Sr. R., Beltran-Przekurat R., Nobis T., and Vaidya S., 2008. Effect of explicit urban land surface representation on the simulation of the 26 July 2005 heavy rain event over Mumbai, India. Atmospheric Chemistry and Physics, 8, 5975 - 5995.

[27] Lei M., and Niyogi D., 2011. Simulation of Indianapolis Thunderstorms using Explicit Urban Parameterization within a Mesoscale Model. Journal of Applied Meteorological Climatology.

[28] Lipp M., Wonka P., and Wimmer M. 2008. Interactive visual editing of grammars for procedural architecture. ACM Transactions on Graphics 27, 3, 1-10.

[29] Masson, V., 2000. A physically-based scheme for the urban energy budget in atmospheric models. Boundary-Layer Meteorology, 94, 357-397.

[30] McCarthy, M. P., Best M. J., and Betts R. A., 2010. Climate change in cities due to global warming and urban effects, Geophys. Res. Lett., 37, L09705.

[31] Niyogi, D., Holt, T., Zhong, S., Pyle, P. C., and Basara, J., 2006. Urban and land surface effects on the 30 July 2003 mesoscale convective system event observed in the Southern Great Plains. Journal of Geophysical Research, 111, D19107.

[32] Niyogi, D., P. Pyle, M. Lei, C. Kishtawal, S. Arya, S. P., Shepherd, M., Chen, F., and Wolfe, B., 2010. Urban modification of thunderstorms: An Observational storm climatology and Modeling case study for the Indianapolis, Urban Region. Journal of Applied Meteorology and Climatology.

[33] Oberndorfer E., Lundholm J., Bass B., Coffman R., Doshi H., Dunnett N., Gaffin S., Kohler M., Liu K.K.Y., and Rowe B., 2007. Green roofs as urban ecosystems: Ecological Structures, Functions, and Services. BioScience, 57(10), 823-833.

[34] Oke, T. R., 1988. The Urban Energy Balance. Progress in Physical Geography, 12, 471-508.

[35] Oleson K. W., Bonan G. B., and Feddema J., 2010. Effects of white roofs on urban temperature in a global climate model. 
Geophysical Research Letters, 37, L03701, DOI:10.1029/ 2009GL042194.

[36] Parish Y. I. H. and Mueller P., 2001. Procedural modeling of cities. In Proceedings of A CM SIGGRA PH, pp. 301-308.

[37] Pettit C., Raymond C., Bryan B., Lewis H., 2011. Identifying strengths and weaknesses of landscape visualisation for effective communication of future alternatives. Landscape and Urban Planning, 100(3), 231-241.

[38] Portugali J., 2000. Self-Organization and the City. Berlin: SpringerVerlag.

[39] Randall T., Churchill C., Baetz B., 2003. A GIS-based decision support system for neighbourhood greening. Environment and Planning B, 30, $541-563$.

[40] Rosenzweig C., Gaffin S., and Parshall, L., 2006. Green Roofs in the Metropolitan Region Research Report. Columbia University Center for Climate Systems Research, NASA Goddard Institute for Space Studies, New York, New York.

[41] Schwartzman Y., and Borning A., 2007. The indicator browser: A web-based interface for visualizing UrbanSim simulation results. In Hawaii International Conference on System Sciences, 92a.

[42] Shashua-Bar L., Pearlmutter D., Erell E., 2009. The cooling efficiency of urban landscape strategies in a hot dry climate. Landscape and Urban Planning, 92(3-4), 179-186.

[43] Shepherd, J. M., Pierce, H., and Negri, A. J., 2002. Rainfall Modification by Major Urban Areas: Observations from Spaceborne Rain Radar on the TRMM Satellite. Journal Applied Meteorology, 41, 689-701.

[44] Shepherd J. M., Carter M., Manyin M., Messen D., and Burian S., 2010. The impact of urbanization on current and future coastal precipitation: a case study for Houston. Environment and Planning B, 37, 284-304.

[45] Stremke S., Koh J., 2010. Ecological concepts and strategies with relevance to energy-conscious spatial planning and design. Environment and Planning B, 37, 518 - 532.

[46] Teemusk, A. and Mander, U., 2010. Temperature Regime of Planted Roofs Compared with Conventional Roofing Systems. Ecological Engineering, 36 (1), 91.

[47] Terzi F., Kaya H.S., 2011. Dynamic spatial analysis of urban sprawl through fractal geometry: the case of Istanbul. Environment and Planning B, 38, 175 - 190.

[48] Torrens P., and O'Sullivan D., 2001. Cellular automata and urban simulation: where do we go from here? Environment $\mathcal{E}$ Planning B: Planning and Design 28, 2, 163-168.

[49] Vanegas, C.A., Aliaga, D, Benes, B, and Waddell, P., 2009a. Visualization of Simulated Urban Spaces: Inferring Parameterized Generation of Streets, Parcels, and Aerial Imagery. IEEE Transactions on Visualization and Computer Graphics, 15(3), 424435.

[50] Vanegas, C.A., Aliaga, D, Benes, B, and Waddell, P., 2009b. Interactive Design of Urban Spaces using Geometrical and Behavioral Modeling. ACM Transactions on Graphics, 28(5), 10 pp.

[51] Waddell P., 2002. UrbanSim: Modeling urban development for land use, transportation, and environmental planning. Journal of the American Planning Association 68, 3, 297-314.

[52] Weber B., Muller P., Wonka P., and Gross M., 2009. Interactive geometric simulation of $4 \mathrm{D}$ cities. Computer Graphics Forum (Proceedings of Eurographics) 28, 2.

[53] Westervelt J., BenDor T., Sexton J., 2011. A technique for rapidly forecasting regional urban growth. Environment and Planning $B, 38,61-81$. 